\title{
On the origin of the FeK $\alpha$ line in the Seyfert 2 galaxy NGC 7172
}

\author{
M. Dadina ${ }^{1,2,3}$, L. Bassani ${ }^{3}$, M. Cappi ${ }^{3}$, G. G. C. Palumbo ${ }^{4}$,L. Piro ${ }^{2}$, M. Guainazzi ${ }^{5}$, G. Malaguti ${ }^{3}$, \\ G. Di Cocco ${ }^{3}$, and M. Trifoglio ${ }^{3}$ \\ 1 BeppoSAX SOC, Telespazio, via Corcolle 19, 00131 Roma, Italy \\ 2 Istituto di Astrofisica Spaziale, CNR, via Fosso del Cavaliere, 00133 Roma, Italy \\ 3 Istituto Te.S.R.E, CNR, via Gobetti 101, 40129 Bologna, Italy \\ 4 Università degli Studi di Bologna, Dip. di Astronomia, Via Ranzani 1, 40127 Bologna, Italy \\ 5 XMM-Newton SOC, VILSPA - ESA, Apartado 50727, 28080 Madrid, Spain
}

Received 5 April 2000 / Accepted 9 January 2001

\begin{abstract}
The Seyfert 2 Galaxy NGC 7172 was observed twice by BeppoSAX narrow field instruments approximately one year apart. As found in previous observations, the source is variable on short time scales (hours) by a factor of $\sim 30 \%$, confirming the presence of a type 1 nucleus at the center. A strong flux variability (by a factor of 2) on a longer time scale was observed between the two observations. Indeed, in November 1997, the lowest flux ever recorded was detected by BeppoSAX. The broad band spectra obtained with the BeppoSAX narrow field instruments show marginal evidence of a reflection component. This structure could explain the very flat spectra previously observed in the $2-10 \mathrm{keV}$ band. An FeK $\alpha$ line was also detected in both observations. The line intensity appears to remain almost constant between the two observations even if the associated large errors cannot exclude some variability. In order to understand the problem of the origin of the line, data from previous observations, performed with other satellites, were also used. The scenario favored is one where the line is produced at a distance of approximatively 8 light years from the continuum source region, rather than having its origin in the accretion disk. In the framework of the standard unified models for AGN, this suggests that its origin is located in the molecular torus.
\end{abstract}

Key words. X-rays: galaxies - galaxies: Seyfert - galaxies: individual: NGC 7172

\section{Introduction}

NGC 7172 , one of the prototype narrow emission line galaxies (NELGs, Bradt et al. 1978), is a S0-Sa type galaxy $(z=0.00868)$ member of the Hickson's compact group HCG90. The galaxy is seen edge-on and has a prominent equatorial dust lane. Its nuclear optical emission spectrum is indicative of excitation by a power-law photoionization source. The large narrow Balmer line decrement $(\geq 6.5$, Sharples et al. 1984) indicates substantial obscuration in the narrow line region $\left(A_{\mathrm{v}} \sim 2.5 \mathrm{mag}\right.$ or $\left.5.610^{21} \mathrm{~cm}^{-2}\right)$.

The galaxy, first identified as an X-ray source by the HEAO1/A2 experiment (Marshall et al. 1979) is one of the brightest Seyfert 2 galaxies at these energies and one of the Piccinotti sample sources (Piccinotti et al. 1982). It has been the target of various observations in both soft and hard X-rays since its discovery (see Polletta et al. 1996 for a recent compendium of X-ray data).

At soft energies, the location of the source in the compact group complicates the spectral analysis and recent ROSAT data suggest that most of the emission below

Send offprint requests to: M. Dadina,

e-mail: dadina@tesre.bo.cnr.it
$2 \mathrm{keV}$ is indeed due to the intergalactic gas of the group (Guainazzi et al. 1998).

The source is variable in the $2-10 \mathrm{keV}$ range both on short and long time scales (Guainazzi et al. 1998); in particular, after a long period (1977-1995) of fairly constant flux, the source suddenly dimmed by almost a factor of 4 within 5 months. The spectrum in this band is fairly simple, being characterized by a strongly $\left(N_{\mathrm{H}} \sim 810^{22} \mathrm{~cm}^{-2}\right)$ absorbed power-law (photon index $\Gamma=1.5-1.8$ ) and a narrow line at $6.4 \mathrm{keV}$ with an equivalent width of 40-120 eV (Smith \& Done 1996; Turner et al. 1997; Guainazzi et al. 1998).

During the latest ASCA observation (Guainazzi et al. 1998), characterized by a low flux level $\left(\sim 10^{-11} \mathrm{erg} \mathrm{cm}^{-2} \mathrm{~s}^{-1}\right)$, the spectrum was flatter and the line equivalent width $(E W)$ higher than in previous observations. The $E W$ increase was consistent either with a change in the continuum and line level or in the continuum flux only. Although the Ginga data gave some evidence for the presence of a reflection component (Nandra \& Pound 1994; Smith \& Done 1996), which could also explain the flat spectrum observed at low flux levels, this issue is still debatable. Alternatively, the flat spectrum could be due to 
complex absorption in the source (Guainazzi et al. 1998). NGC 7172 was also observed at high energies both by OSSE and BATSE on CGRO: the spectral photon index at these energies is canonical $\left(\Gamma=2.0_{-0.3}^{+0.4}\right)$ and the flux is typically $7-810^{-11} \mathrm{erg} \mathrm{cm}^{-2} \mathrm{~s}^{-1}$ in the $20-100 \mathrm{keV}$ band (Ryde et al. 1997; Malizia et al. 1999).

Here we present the broad band (0.1-100 keV) spectrum of NGC 7172 obtained by BeppoSAX on two subsequent observations performed approximately one year apart.

\section{Observations and data reduction}

Table 1. BeppoSAX observations log

\begin{tabular}{lcc}
\hline \hline Obs. (date) & Integration Time (ks.) & $1^{-3}$ Counts s $^{-1}$ \\
\hline A (Oct. 15, 1996) & & \\
LECS & 15.2 & $8.9 \pm 0.8$ \\
MECS & 39.2 & $144 \pm 2$ \\
PDS & 17.3 & $338 \pm 36$ \\
\hline B (Nov. 6, 1997) & & \\
LECS & 22.5 & $6.1 \pm 0.5$ \\
MECS & 49.4 & $51 \pm 1$ \\
PDS & 21.1 & $199 \pm 33$ \\
\hline \hline
\end{tabular}

The BeppoSAX X-ray observatory (Boella et al. 1997) is a major program of the Italian Space Agency with participation of the Netherlands Agency for Aerospace Programs and ESA. The work presented here concerns observations performed with the Narrow Field Instruments (NFI) on-board the satellite: the Low Energy Concentrator Spectrometers (LECS, covering the 0.1-10 keV band; Parmar et al. 1998), the Medium Energy Concentrator Spectrometer (MECS, covering the 1-10.5 keV band; Boella et al. 1997), the High Pressure Gas Scintillation Proportional Counter (HPGSPC covering the 4-120 keV band; Manzo et al. 1997) and the Phoswich Detection System (PDS, covering the 15-200 keV band; Frontera et al. 1997).

BeppoSAX NFI pointed at NGC 7172 on October 15th, 1996 (observation A) and re-observed the source on November 6th, 1997 (observation B) because the first pointing was interrupted by a gamma-ray burst TOO. The second observation was performed with only MECS units 2 and 3 since the unit 1 failed between the two pointings.

The effective exposure times and net count rates for each narrow field instrument are listed in Table 1 for both BeppoSAX observations.

For the data reduction and analysis, performed using ftools 4.1 and XANADU 10.0 software packages, we used standard techniques and criteria. Light curves and spectra were extracted from a region centered on NGC 7172 and having radius of $8^{\prime}$ for LECS and $4^{\prime}$ for MECS.

Figures 1 and 2 show the light curves in the $2-10 \mathrm{keV}$ band for both observations. While during observation $\mathrm{A}$

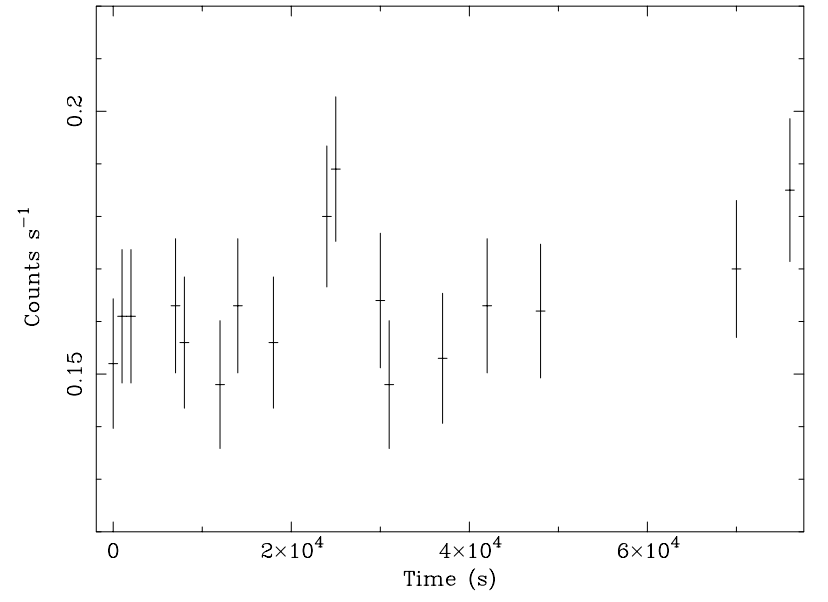

Fig. 1. MECS (2-10 keV) light curve of observation A (using a time bin of $1000 \mathrm{~s}$ )

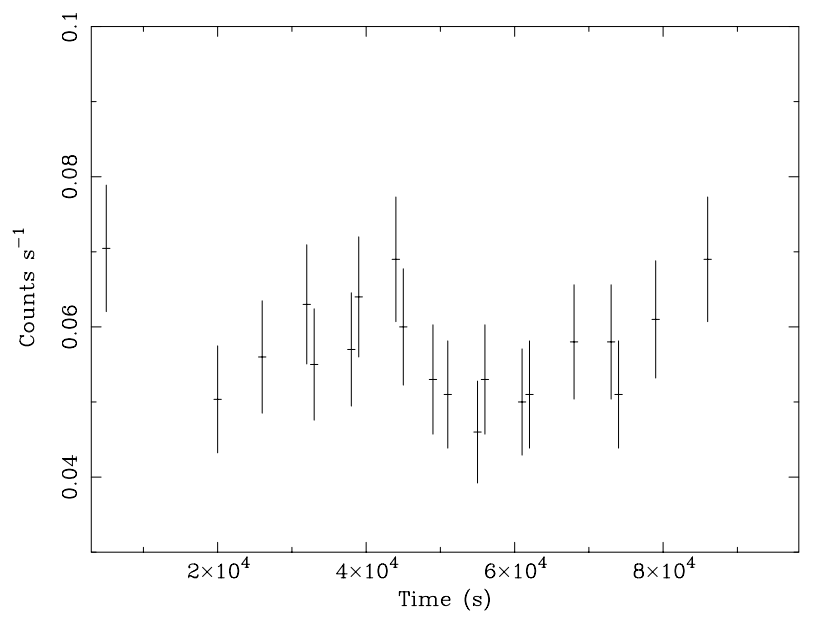

Fig. 2. MECS (2-10 keV) light curve of observation B (using a time bin of $1000 \mathrm{~s}$ )

the source intensity was stable except for a rapid rise and decline of $20-25 \%$ at $t \sim 2.510^{4} \mathrm{~s}$, during observation $\mathrm{B}$ NGC 7172 showed a systematic variability of $\sim 30 \%$ similar to that detected in the last ASCA observation (Guainazzi et al. 1998). This lead us to conclude that a Seyfert 1like nucleus is present in NGC 7172. Due to the limited statistics available, we find no significant spectral variations and therefore the source data were integrated over the entire observation to obtain average spectra.

The LECS field is certainly contaminated by the soft $\mathrm{X}$-ray emission from the embedding compact group, while the hard X-ray band (MECS) is dominated by NGC 7172. No bright sources emitting in the $2-10 \mathrm{keV}$ band are known to be present in the PDS field of view of 1.3 degrees $(F W H M)$; the BL Lac object PKS 2155-304 is 1.8 degrees away from NGC 7172, far enough to exclude contamination of the high energy emission.

The LECS background spectrum was extracted from two semi annuli in the instrument field of view which were then normalized to the source extraction region as described in Parmar et al. (1999). The MECS background subtraction was instead performed by means of blank 
sky deep field exposures accumulated by the BeppoSAX Science Data Center at the beginning of the mission. The PDS reduction was performed using the SAXDAS software package taking into account both rise-time and spike corrections. Products were obtained by plain subtraction of the "off" from the "on" source data. No HPGSPC data will be considered in the present paper as the source is too faint for a correct background subtraction.

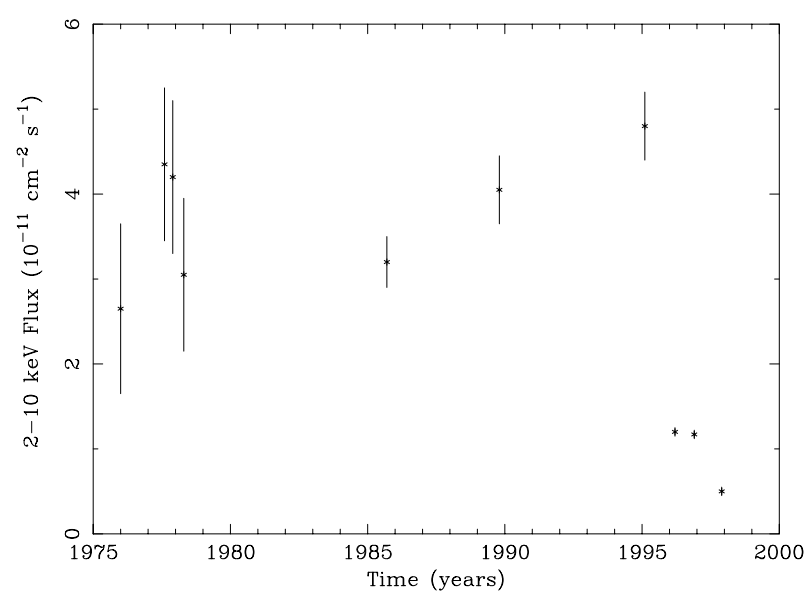

Fig. 3. The long-term NGC 7172 light curve. Data are from the Ariel V observation (1976), the HEAO-1/A2 observations (1977.6, 1977.9, 1978.3), the EXOSAT observation (1985.7), the Ginga observation (1989.8), the ASCA observations $(1995.5,1996.5)$ and the BeppoSAX observations (1996.7, 1997.8). The source flux declines by a factor $\sim 10$ from the 1995 ASCA observation to the 1997 BeppoSAX observation, during which the source was detected at the lowest state ever recorded

LECS and MECS data were rebinned in order to sample the energy resolution of the detector with an accuracy proportional to the count rate; furthermore, we ensure at least 20 counts per channel which allows applicability of the $\chi^{2}$ statistics.

\section{Spectral analysis}

Spectral data from LECS, MECS and PDS were fitted simultaneously. We restricted the spectral analysis to the 0.12-4.0 keV range for LECS and $1.65-10 \mathrm{keV}$ energy bands for MECS where the response matrices released in September 1997 are best calibrated; PDS analysis was restricted to data below $90 \mathrm{keV}$ as the source signal disappears above this energy. Normalization constants were introduced to allow for known differences in the absolute cross-calibration between detectors. The values of the two constants were allowed to vary. The MECS-LECS constant always turned out to be within $10 \%$ of the suggested value of 0.9 (see Fiore et al. 1999 for a discussion). The MECS-PDS constant was constrained to vary within the suggested 0.7-0.9 interval (Fiore et al. 1999).

All quoted errors correspond to $90 \%$ confidence intervals for one interesting parameter $\left(\Delta \chi^{2}=2.706\right)$. All models used in what follows contain an additional term

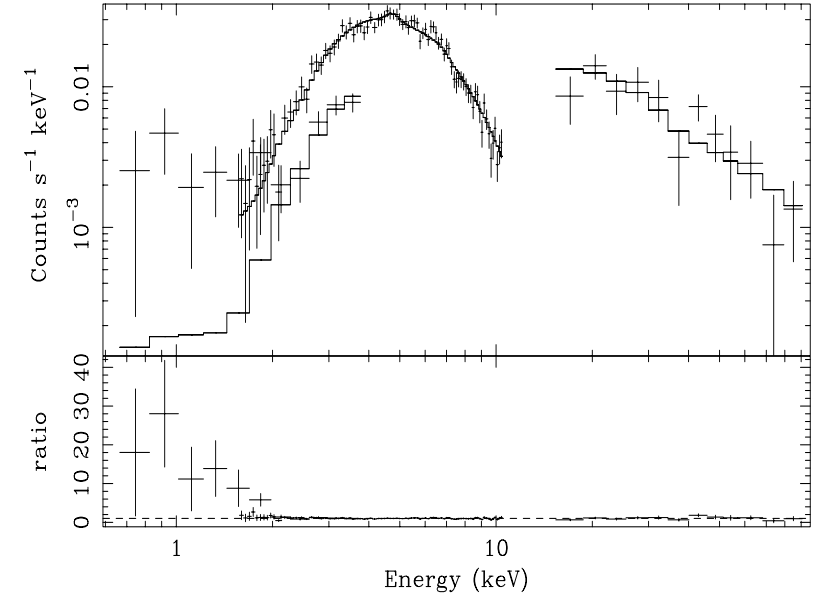

Fig. 4. The 0.6-90 keV BeppoSAX data of observation A modeled with a simple absorbed power-law (upper panel). In the lower panel the ratio between the data and model is plotted. A huge soft excess is clearly visible at low energies extending from 0.6 up to $\sim 2.0 \mathrm{keV}$. Very similar results are obtained for observation B

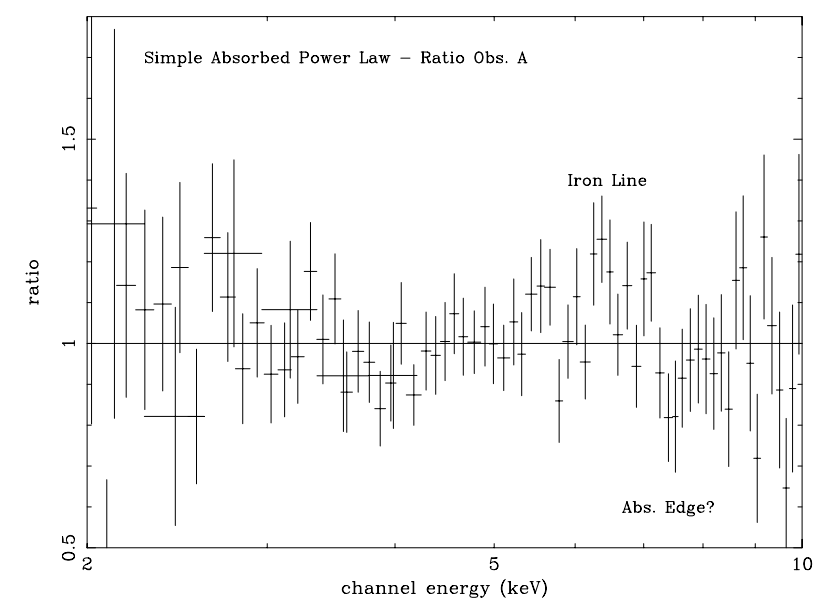

Fig. 5. The ratio between the data and the simple power-law model in the $2-10 \mathrm{keV}$ band for observation A. The features due to the FeK $\alpha$ fluorescent line (at $6.4 \mathrm{keV}$ ) and, possibly, an absorption edge (at $7.1 \mathrm{keV}$ ) are visible

to allow for the absorption of X-rays due to our Galaxy which, in the direction of NGC 7172 , is $1.6510^{20} \mathrm{~cm}^{-2}$ (Dickey \& Lockman 1990).

During observation A, the $2-10 \mathrm{keV}$ flux was $\sim 1.210^{-11} \mathrm{erg} \mathrm{cm}^{-2} \mathrm{~s}^{-1}$, i.e. similar to what was observed in the ASCA observation performed only 5 months earlier. In observation B, the flux decreased to $\sim 510^{-12} \mathrm{erg} \mathrm{cm}^{-2} \mathrm{~s}^{-1}$, indicating that the long trend of intensity decline (see Fig. 3) firstly observed by ASCA in the 1996 observation, was still persisting a year later.

The broad band spectra were first fitted with a simple absorbed power-law (Fig. 4) to highlight any extra features. The most prominent deviation from an absorbed power-law is a huge soft excess below $\sim 2.0 \mathrm{keV}$. Due to the low statistic available in this energy range, no detailed investigation about this band of the spectrum is possible. Nevertheless, following Ponman et al. (1996) 
Table 2. Power-law models

\begin{tabular}{|c|c|c|c|c|c|c|}
\hline Obs. $^{a}$ & Mod. & $N_{\mathrm{H}}^{b}$ & $\Gamma^{c}$ & $E_{\text {Line }}^{d}$ & $I_{\text {Line }}^{e}$ & $\chi^{2} /$ d.o.f \\
\hline & & $10^{22} \mathrm{~cm}^{-2}$ & & $\mathrm{keV}$ & $10^{-5}$ ph. $\mathrm{cm}^{-2} \mathrm{~s}^{-1}$ & \\
\hline \multirow[t]{2}{*}{ A } & abs. power-law & $10.21_{-0.79}^{+0.80}$ & $1.70_{-0.12}^{+0.12}$ & & & $86.16 / 84$ \\
\hline & abs. power-law + Gaussian line & $9.84_{-0.71}^{+0.76}$ & $1.67_{-0.08}^{+0.09}$ & $6.46_{-0.15}^{+0.16}$ & $2.39_{-1.29}^{+1.28}$ & $77.14 / 82$ \\
\hline \multirow[t]{2}{*}{ B } & abs. power-law & $9.53_{-1.27}^{+1.38}$ & $1.62_{-0.14}^{+0.24}$ & & & $72.33 / 64$ \\
\hline & abs power-law + Gaussian line & $9.01_{-1.28}^{+1.38}$ & $1.60_{-0.13}^{+0.14}$ & $6.46_{-0.13}^{+0.15}$ & $2.18_{-1.08}^{+1.08}$ & $61.47 / 62$ \\
\hline
\end{tabular}

$\left({ }^{a}\right)$ BeppoSAX observation, $\left({ }^{b}\right)$ absorbing column density, $\left({ }^{c}\right)$ photon index, $\left({ }^{d}\right)$ line energy, $\left({ }^{e}\right)$ line intensity.

Table 3. Power-law + reflection component + Gaussian line

\begin{tabular}{|c|c|c|c|c|c|c|}
\hline Obs. ${ }^{a}$ & $N_{\mathrm{H}}^{b}$ & $\Gamma^{c}$ & $E_{\text {Line }}^{d}$ & $I_{\text {Line }}{ }^{e}$ & $R^{f}$ & $\chi^{2} /$ d.o.f \\
\hline & $10^{22} \mathrm{~cm}^{-2}$ & & $\mathrm{keV}$ & $10^{-5}$ ph. $\mathrm{cm}^{-2} \mathrm{~s}^{-1}$ & & \\
\hline A & $10.18_{-0.78}^{+0.80}$ & $1.91_{-0.21}^{+0.23}$ & $6.45_{-0.33}^{+0.21}$ & $1.72_{-1.50}^{+1.40}$ & $2.24_{-1.61}^{+3.96}$ & $73.64 / 81$ \\
\hline B & $9.82_{-1.41}^{+1.38}$ & $1.91_{-0.34}^{+0.41}$ & $6.47_{-0.15}^{+0.20}$ & $1.92_{-1.21}^{+1.16}$ & $2.11_{-1.95}^{+0.97}$ & $58.61 / 61$ \\
\hline
\end{tabular}

$\left({ }^{a}\right)$ BeppoSAX observation, $\left({ }^{b}\right)$ absorbing column density, $\left({ }^{c}\right)$ photon index, $\left({ }^{d}\right)$ line energy, $\left({ }^{e}\right)$ line intensity, $\left({ }^{f}\right)$ relative reflected-direct normalization.

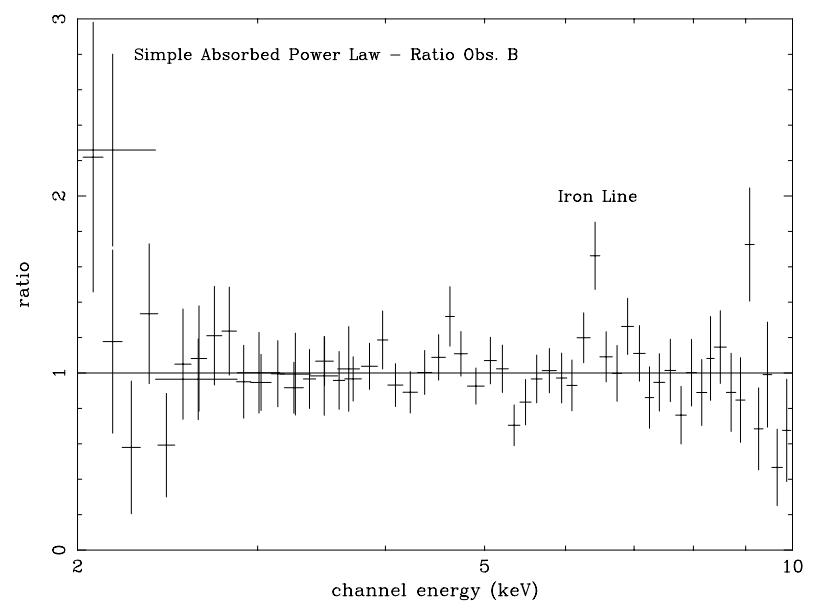

Fig. 6. The ratio between the data and the simple power-law model in the $2-10 \mathrm{keV}$ band for observation B. A soft excess extending in the $2-2.5 \mathrm{keV}$ band is visible

and Guainazzi et al. (1998), we tried to fit the excess with a thermal plasma model (namely the MEKAL model in xspec, Mewe, Gronenschild \& van der Oord 1985;
Mewe \& van der Oord 1986; Kaastra 1992; Arnaud \& Rothenflug 1985), adopting, for energies $\geq 2 \mathrm{keV}$, the best fit model discussed in the next section. The absorbing column density associated with this component was fixed to the galactic value while the metal abundance was assumed to be 0.2 as obtained by ROSAT (Ponman et al. 1996). This model provides a best fit temperature of $0.9 \pm 0.2 \mathrm{keV}$ and $1.2 \pm 0.5 \mathrm{keV}$ for observation $\mathrm{A}$ and $\mathrm{B}$ respectively. These values are, within the errors, in agreement with the temperature measured by ASCA $(0.68 \pm 0.12 \mathrm{keV}$, Guainazzi et al. 1998). As already suggested, this soft component is probably due to the thermal emission of the gas in the compact group.

\subsection{Emission in the $2-90 \mathrm{keV}$ energy range}

To avoid contamination from the compact group diffuse emission, we restricted the spectral analysis of the nuclear emision of NGC 7172 to data above $2 \mathrm{keV}$ for observation $\mathrm{A}$ and $2.5 \mathrm{keV}$ for observation $\mathrm{B}$. We expect that the effects of the thermal plasma associated with HCG90 are negligible at these energies. 
Fitting the data in these restricted energy bands with an absorbed power law model provides a column density of $\sim 10^{23} \mathrm{~cm}^{-2}$ and a photon index of $1.6-1.7$ (Table 2). The photon index is in agreement with the values obtained for samples of Seyfert galaxies observed both by Exosat and Ginga $(\Gamma \sim 1.7-1.8$, Turner \& Pounds 1989; Nandra \& Pounds 1994) but it is only marginally consistent with what was obtained in the ASCA 1995 and 1996 observations, when the source showed a very flat spectrum ( $\Gamma \sim 1.5$, Ryde et al. 1997; Guainazzi et al. 1998).

Inspection of Figs. 5 and 6 , where the ratio between the data and the absorbed power-law model is plotted, indicates the presence of an $\mathrm{FeK} \alpha$ line and possibly (in observation A) an absorption edge in the $6-8 \mathrm{keV}$ band.

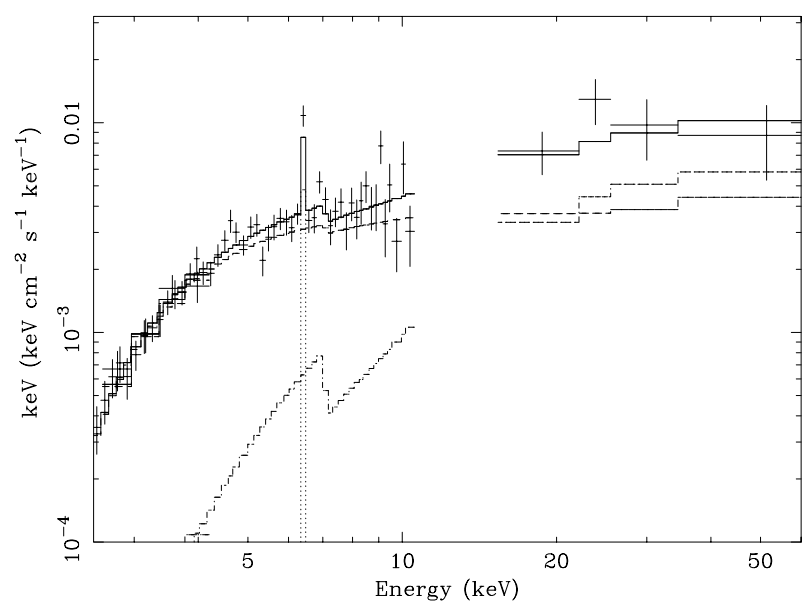

Fig. 7. BeppoSAX spectrum of NGC 7172 (observation B) fitted with a power-law plus a reflection component and a narrow Gaussian line (PDS data have been rebinned so as to have $S / R$ $\geq 3$ for clarity)

The addition of a narrow line to the absorbed powerlaw model provides an improvement in the quality of the fit by a $\Delta \chi^{2} \sim 9.0$ for two extra degrees of freedom for both observations $\mathrm{A}$ and $\mathrm{B}$, implying that the line is required at more than $99.9 \%$ confidence level (Table 2). The line is located at $6.46 \pm 0.20 \mathrm{keV}$ and has an equivalent width $(E W)$ of $120 \pm 65 \mathrm{eV}$ in the first observation, increasing to $210 \pm 105 \mathrm{eV}$ in the second one. If the line width is allowed to vary, the additional parameter does not give a significant improvement in the quality of the fit and yields an upper limit to the Gaussian width of $\sigma \leq 0.75 \mathrm{keV}$, consistent with the energy resolution of the MECS ( $7 \% F W H M$ at $\sim 6.4 \mathrm{keV}$, Piro et al. 1995; Boella et al. 1997). Due to the available statistics, the line width was thus frozen to zero in the subsequent analysis.

Due to the possible presence of an absorption edge in observation A (no similar feature is present in observation B), we added this component to the previous model. The energy of the edge was found to be $7.3 \pm 0.5 \mathrm{keV}$ but it was not statistically significant $\left(\Delta \chi^{2}=3.8\right.$ for 2 more parameters of interest) giving an optical depth $\tau \leq 0.4$

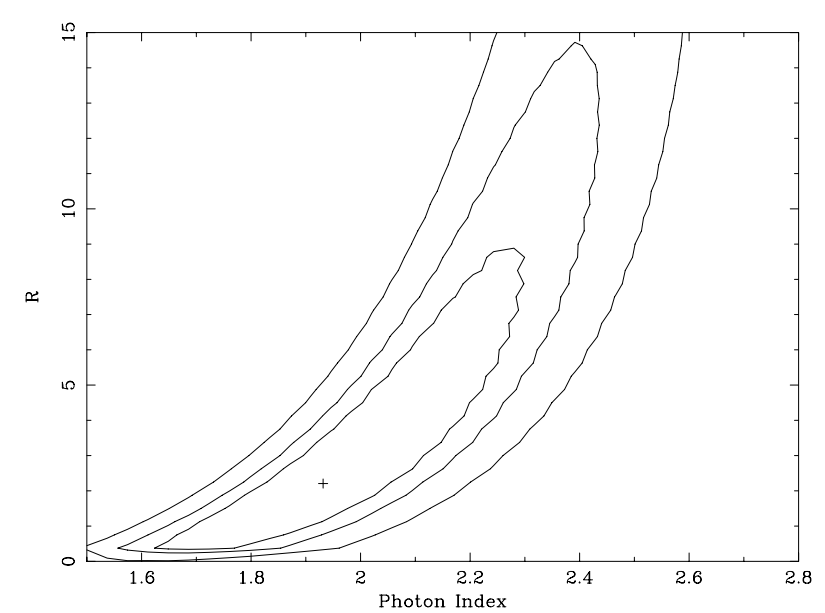

Fig. 8. The relative reflected-direct normalization $(R)$ vs. the photon index $\Gamma$ contour plot for observation B. The contours are at 68,90 and $99 \%$ confidence levels going from the inner to the outer. The model is acceptable with a confidence level of $90 \%$. A completely similar plot is obtained for observation A

$\left(\tau=N_{\mathrm{H}} \times \sigma(E)\right.$ where $\sigma(E)$ is the photoelectric cross section).

Since Guainazzi et al. (1998) used a dual absorber model to explain the flat spectrum for the ASCA 1996 data, we tried a similar model. The quality of the data prevents us from constraining the values of the model parameters if they are allowed to vary. For this reason and also for comparison with the ASCA 1996 results, the power-law photon index was fixed to 1.9 (as obtained by Guainazzi et al. 1998) and the FeK $\alpha$ line energy was frozen to $6.4 \mathrm{keV}$. We obtained a slight improvement in the fit and the two column densities became $10^{23} \mathrm{~cm}^{-2}$ for the total absorber and $610^{23} \mathrm{~cm}^{-2}$ for the partial absorber with a covering fraction of $\sim 30 \%$. However, again there was no significant improvement in the fit and we had no evidence either for a flat spectrum as observed by ASCA in 1996 or for an absorption edge. We, therefore, do not consider this model necessary for our data and do not discuss it further.

Instead, we consider the possible presence in NGC 7172 of a reprocessed component due to reflection in an optically thick cold medium (Table 3 ). This is a common feature in Seyfert galaxies and we used the pexrav model in xspec (Magdziarz \& Zdziarski 1995) to model it. Due to the poor statistics, we fixed the inclination angle of the reflector to be $60 \mathrm{deg}$, a value which is expected to be suitable for the Seyfert 2 galaxies in the framework of the standard unified models. When this component is added to the absorbed power-law plus emission line model, the fit improves by $\Delta \chi^{2}=3.5$ for observation A and 2.9 for observation B (Fig. 7) for only one more extra parameter of interest. An F-test indicates that this component is required with a confidence level higher than $90 \%$ for both observations. Evidence for the presence of a reflected component in the continuum was also found in Ginga data. The $R$ parameter of the fit, that indicates the relative amount of reflection compared to the directly-viewed 
primary power-law, is only poorly constrained (Fig. 8) giving a best fit value of $\sim 2$ for both observations. It is not clear if the reflection component is an artifact of the fitting procedure. Given the low statistics, we cannot exclude that this strong reflection is, at least in part, due to an artifact of the fitting procedure (namely, the interplay of $R$ with the MECS-PDS cross calibration factor). Assuming that it is indeed real and that the accretion disk is actually the reflector, we would expect a value of $R$ constant in time (since the disk reflection would follow the continuum variations). This is consistent with the fit results (Table 3). However, associated with this component, we would also expect to see a FeK $\alpha$ line with constant $E W \sim 300 \mathrm{eV}$ (George \& Fabian 1991). As will be discussed in the next section, this is not the case for NGC 7172, at least for observation A. On the contrary, assuming that the reflection component is produced far from the direct continuum emission region, we would expect an increase of the parameter $R$ in association with a reduction of the source luminosity, but the quality of the data prevents us from verifying this point. Also, considerations of the photon index $\Gamma$ for the simple absorbed power-law model are not of much help. The spectrum should harden from observation A to observation B but again this is only marginally observed (see Table 2). A possible indication of the absence of this structure in the source's spectrum comes from the RXTE observations of NGC 7172 that do not require reflection to fit the data well. When this component is added to a simple absorbed power-law plus Gaussian line model, a marginal improvement of the fit is obtained with $\Delta \chi^{2} \sim 0.5-1.0$ for one more extra parameter (Georgatopoulos \& Papadakis 2000).

Finally, it is worth noting that, whatever the model used, the absorbing column density turns out to be $N_{\mathrm{H}} \sim$ $10^{23} \mathrm{~cm}^{-2}$, well in agreement with what has been observed previously. This implies that the state of the absorber is not affected by the state of the source.

\subsection{The FeK $\alpha$ line}

The FeK $\alpha$ line in NGC 7172 was first detected by ASCA in the 1995 observation (Ryde et al. 1996; Guainazzi et al. 1998), while a previous Ginga observation in 1989 provided only an upper limit of $\sim 40 \mathrm{eV}$; BeppoSAX detected the line in both observations. Fitting the continuum with an absorbed power-law, we obtain an equivalent width of $120 \pm 65$ and $210 \pm 105 \mathrm{eV}$ for observation A and B respectively, further indicating that the line $E W$ increases as the source flux decreases.

Figure 9 shows the MECS PHA ratio between the two BeppoSAX observations (B/A). In the plot, no significant feature is present at the energy of the $\mathrm{FeK} \alpha$ line. At first glance, this would imply that the line follows the changes of the continuum flux. However, a more careful inspection indicates that the absence of any significant feature in the PHA ratio is possibly due to our limited statistics. Assuming that the line flux was constant between the two

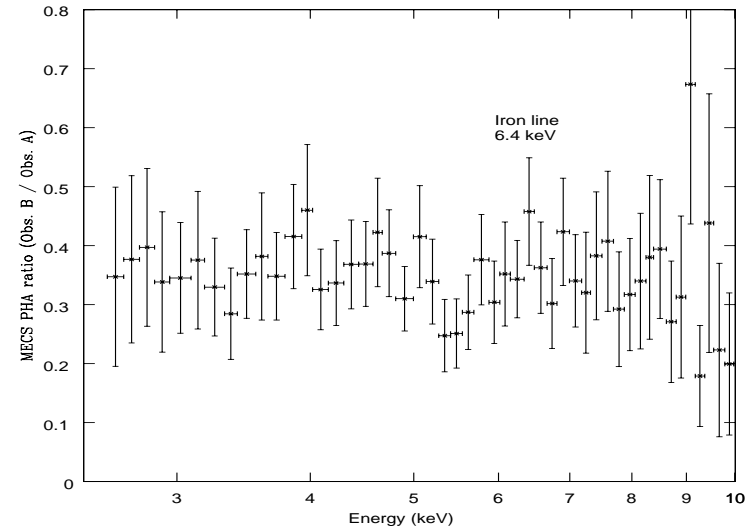

Fig. 9. MECS PHA ratio between the two BeppoSAX observations $(\mathrm{B} / \mathrm{A})$. No significant excess is visible at the energy of the $\mathrm{FeK}_{\alpha}$ line

observations, we predict a $\sim 10 \%$ feature in the PHA ratio, i.e. an undetectable excess considering the statistics available.

To further investigate the issue of the $\mathrm{FeK}_{\alpha}$ line variability, we reanalyzed the old Ginga and ASCA observations of NGC 7172 in order to estimate the order of magnitude of the changes involved.

All the data were fitted with a simple absorbed power law plus FeK $\alpha$ line so as to have uniformity in the treatment of the continuum modeling (note that the reflection component is not required by the ASCA data). In Fig. 10 the line flux is plotted against the $1 \mathrm{keV}$ continuum normalization, while in Fig. 11 the line equivalent width is shown as a function of the $2-10 \mathrm{keV}$ flux.

If the line originates in the accretion disk, then the change by a factor of $\sim 10$ in the continuum level (Fig. 10), would produce a change of an equal amount in the line intensity, thus providing a constant $E W$ in time.

Instead, we observe a decrease in the line intensity of no more than a factor of $\sim 7$ (this value was obtained in Fig. 10 considering the $90 \%$ confidence contours). Moreover, Fig. 11 shows an anti-correlation between the line $E W$ and the $2-10 \mathrm{keV}$ flux. We can therefore conclude that the data are not compatible with a pure disk origin of the line.

If instead the line is produced in the absorber itself, assuming a spherical distribution, we would expect an $E W \sim 70 \mathrm{eV}$ for the observed $N_{\mathrm{H}}$ (Makishima 1986). Otherwise, in the framework of the unified models for AGN (Antonucci \& Miller 1985; Antonucci 1993) a molecular torus seen almost edge-on, with the observed $N_{\mathrm{H}}$, would produce an FeK $\alpha$ line with an $E W$ of $30-40 \mathrm{eV}$ (Ghisellini et al. 1994). This value is in perfect agreement with what was measured by Ginga (Nandra \& Pounds 1994) and during the 1995 ASCA observation (Ryde et al. 1996, 1997) when the source was bright but somewhat lower than observed by BeppoSAX. The agreement could, however, be recovered and the anti-correlation in Fig. 11 could be explained if there is a time lag between the continuum and the line production region: as the flux level 


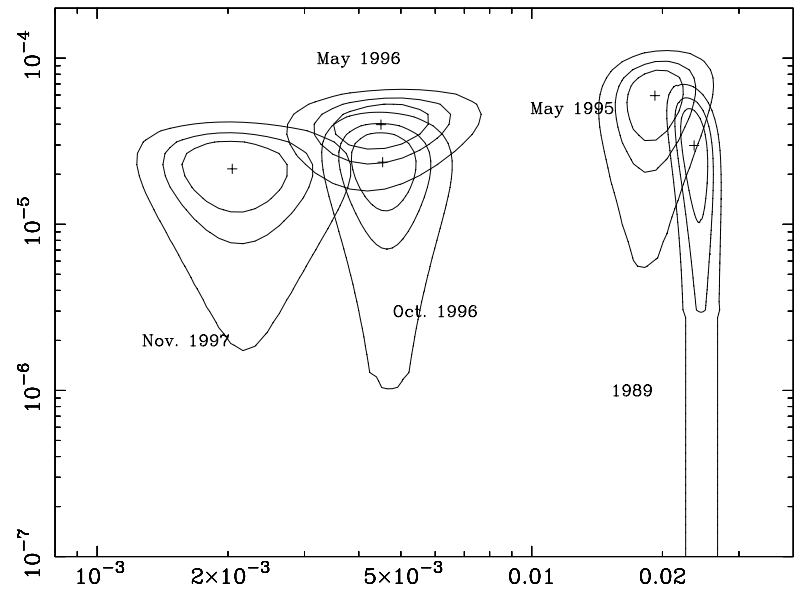

Fig. 10. Contours of the line flux (units of ph. $\mathrm{cm}^{-2} \mathrm{~s}^{-1}$ ) against the power-law normalization (units of ph. $\mathrm{keV}^{-1}$ $\mathrm{cm}^{-2} \mathrm{~s}^{-1}$ at $1 \mathrm{keV}$ ). The 1989 data are from Ginga, May 1995 and 1996 are from ASCA. October 1996 and November 1997 are the BeppoSAX data presented in this paper

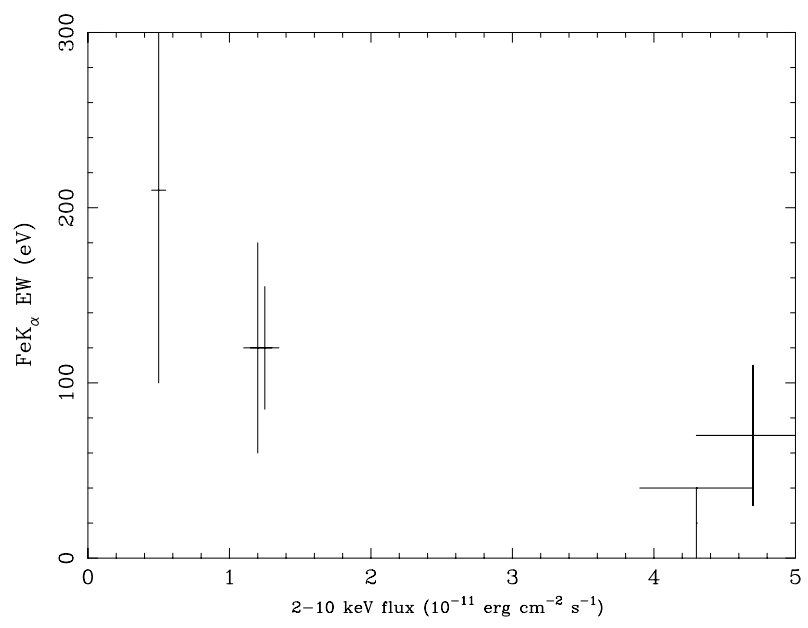

Fig. 11. The FeK $\alpha$ line $E W$ against the $2-10 \mathrm{keV}$ flux during the last 9 years' observations (data are, from left to right, from BeppoSAX 1997, BeppoSAX 1996, ASCA 1996, Ginga 1989, ASCA 1995)

of the nucleus decreases, the line intensity follows these changes with a delay directly proportional to the distance between the continuum production region and the reprocessor. The overall effect is that the line $E W$ increases as the source weakens. Assuming this simplified model we have the opportunity to calculate a lower limit for the distance between the emitter and the reprocessor which is of the order of $\sim 8$ light years for NGC $7172(d \leq c \times \Delta t$ where $d$ is the linear dimension, $c$ is the speed of light and $\Delta t$ is the observed time scale of the variations).

\section{Discussion and conclusions}

We analyzed two BeppoSAX observations, performed in October 1996 and November 1997, of the sky region containing NGC 7172. The source is detected embedded in diffuse soft-X emission from the compact group HCG90, of which the Seyfert 2 is a member.

NGC 7172 showed flux variability in the $2-10 \mathrm{keV}$ range. During observation $B$ the source flux varied by a factor of $\sim 30 \%$ thus indicating the presence of a type $1 \mathrm{nu}-$ cleus in its center, while the luminosity decreased by a factor of $\sim 2$ between the two BeppoSAX pointings. Indeed, we detected the lowest flux level ever recorded for this source in the 2-10 keV band. During the November 1997 pointing, the source was $\sim 10$ times weaker than when observed a decade before.

A good fit to the data of both observations in the whole 2-90 keV range is yielded by a simple power-law plus Gaussian line model, photoelectrically absorbed by a substantial column density of cold matter. The flat observed spectrum $(\Gamma=1.6-1.7)$ could be due to the presence of a Compton reflection component. The presence of this component is indeed required by the fit at a more than $90 \%$ confidence level, if we want the intrinsic powerlaw index to be equal to the canonical value, 1.9 , observed in Seyfert galaxies. In this case, the amount of reflection is constrained only in the range $\sim 1-3$ (weighted mean of the BeppoSAX measurements). However, the systematics introduced by the uncertinities in the relative normalization between MECS and PDS are likely to substantially affect this (Fiore Guainazzi \& Grandi 1999).

The absorbing column density is $N_{\mathrm{H}} \sim 10^{23} \mathrm{~cm}^{-2}$ (independent of the model adopted for the description of the continuum), as previously observed. This value seems to have remained stable over a $\sim 20$ years period (Malizia et al. 1997; Turner \& Pounds 1989; Smith \& Done 1996; Guainazzi et al. 1998) although the source has dimmed by a factor of $\sim 10$.

A complex absorption model was also tested in order to check if this is the cause of the flatness of the spectrum. The fit obtained is statistically acceptable but we discarded this model since it is not required by the data.

$\mathrm{X}$-ray observations of Seyfert galaxies support the idea that the primary X-ray continuum is reflected from cold material surrounding the central nucleus (Lightman \& White 1988; Ghisellini et al. 1994); this reprocessing produces an $\mathrm{FeK} \alpha$ line and a reflection component. Whether this occurs in the accretion disk or in other structures of the nuclear region (for example the molecular torus of the unified models for AGN) is still debatable for Seyfert 2 galaxies. Given the large amplitude of NGC 7172 continuum variations seen in the last 10 years, it is possible to tackle this issue in this particular case, at least for the FeK $\alpha$ line. We therefore searched the data for evidence of possible changes in the line parameters so as to understand its origin and location.

The pure disk origin of the line is ruled out from the present data for two main reasons: 1) the line intensity does not follow the continuum instantly; 2) the equivalent widths obtained by Ginga $(\leq 40 \mathrm{eV})$ and ASCA 1995 observations $(\sim 60 \mathrm{eV})$ are lower than expected $(E W \sim$ $100-200 \mathrm{eV}$ ) from a disk in a face-on configuration. To reconcile these low $E W$ values with a pure disk origin, 
one should assume that the disk is highly inclined. In this case we expect that the absorber would contribute (with the $N_{\mathrm{H}} \sim 10^{23} \mathrm{~cm}^{-2}$ seen in NGC 7172) by a similar amount to the line $E W$ (Makishima 1986; Ghisellini et al. 1994), overproducing what is observed.

We therefore conclude that the FeK $\alpha$ line is most likely produced far from the continuum source and that it probably emerges from the absorber itself. Here the fluorescent line would be produced via transmission in an optically thin medium. In this case no reflection component would be associated with the $\mathrm{FeK} \alpha$, in agreement with the present measurements. The time lag between the continuum changes and the line reaction allows us to infer the size of the absorber, which is of the order of $\sim 8$ light years.

We speculate that such an absorber can be associated with a parsec-scale molecular torus, as usually assumed in standard unified models for AGN (Antonucci \& Miller 1985; Antonucci 1993). This interpretation would also be in agreement with the $E W$ measured by Ginga and ASCA (Smith \& Done 1996; Guainazzi et al. 1998).

After the present work was completed, we became aware of a paper discussing the same BeppoSAX sets of data by Akylas et al. (2000). The spectral fits obtained by Akylas et al. (2000) are consistent with the present results, although the authors prefer to interpret the data in other terms.

Acknowledgements. The authors want to thank the anonymous referee for his/her helpful comments and patience. This work has been partially supported by the Italian Space Agency (ASI).

\section{References}

Akylas, A., Georgantopoulos, I., \& Comastri, A. 2000, MNRAS, to appear [astro-ph/0101079]

Antonucci, R. R. J. 1993, ARA\&A, 31, 473

Antonucci, R. R. J., \& Miller, J. S. 1985, ApJ, 297, 621

Arnaud, M., \& Rothenflug, M. 1985, A\&AS, 60, 425

Boella, G., Butler, R. C., Perola, G. C., et al. 1997, A\&AS, 122,299

Boella, G., Chiappetti, L., Conti, G., et al. 1997, A\&AS, 122, 327

Bradt, H., \& Margon, B. 1978, S\&T, 56, 499

Dickey, J. M., \& Lockman, F. J. 1990, ARA\&A, 28, 215

Fiore, F., Guainazzi, M., \& Grandi, P. 1999, Handbook for NFI Spectral Analysis, http://www.sdc.asi.it/software/

Frontera, F., Costa, E., dal Fiume, D., et al. 1997, SPIE, 3114, 206
Georgantopoulos, I., Papadakis, I., MNRAS, submitted [astro-ph/0008295]

George, I. M., \& Fabian, A. C. 1991, MNRAS, 267, 743

Ghisellini, G., Haardt, F., \& Matt, G. 1994, MNRAS, 267, 743

Guainazzi, M., Matt, G., Antonelli, L. A., et al. 1998, MNRAS, 298,824

Haardt, F., \& Maraschi, L. 1993, ApJ, 413, 507

Haardt, F., Maraschi, L., \& Ghisellini, G. 1994, ApJL, 432, L95

Kaastra, J. S. 1992, An X-Ray Spectral Code for Optically Thin Plasmas, Internal SRON-Leiden Report, updated version 2.0

Lightman, A. P., \& White, T. R. 1988, ApJ, 335, 57

Magdziarz, P., \& Zdziarski, A. A. 1995, MNRAS, 273, 837

Makishima, K. 1986, in Proc. of the conference: The Physics of Accretion onto Compact Objects, ed. K. O. Mason, M. G. Watson, \& N. E. White, Tenerife, Lect. Notes Phys., 266, 249

Malizia, A., Bassani, L., Zhang, S. N., et al. 1999, ApJ, 519, 637

Manzo, G., Giarrusso, S., Santangelo, A., et al. 1997, A\&AS, 122,341

Marshall, F. E., Boldt, E. A., Holt, S. S., et al. 1979, ApJS, 40, 657

Mewe, R., Gronenschild, E. H. B. M., \& van der Oord, G. H. J. 1985, A\&AS, 62, 197

Mewe, R., Lemen, J. R., \& van der Oord, G. H. J. 1986, A\&AS, 65,511

Nandra, K., \& Pounds, K. A. 1994, MNRAS, 268, 405

Parmar, A. N., Bavdaz, M., Favata, F., et al. 1998, IAU Symp. 188, The Hot Universe, 188, 71

Parmar, A. N., Oosterbroek, T., Orr, A., et al. 1999, A\&AS, 136,407

Piccinotti, G., Mushotzky, R. F., Boldt, E. A., et al. 1982, ApJ, 253,485

Piro, et al. 1995, BeppoSAX Observer Handbook http://www.sdc.asi.it/

Polletta, M., Bassani, L., Malaguti, G., Palumbo, G. G. C., \& Caroli, E. 1996, ApJS, 106, 399

Ponman, T. J., Bourner, P. D. J., Ebeling, H., \& Böhringer, H. 1996, MNRAS, 283, 690

Ryde, F., Poutanen, J., Svensson, R., et al. 1996, in Proc. of the conference: X-Ray Imaging and Spectroscopy of Cosmic Hot Plasmas, ed. F. Makino, \& K. Mitsuda, Tokyo, 299

Ryde, F., Poutanen, J., Svensson, R., Larsson, S., \& Ueno, S. 1997, A\&A, 328, 69

Sharples, R. M., Longmore, A. J., Hawarden, T. G., \& Carter, D. 1984, MNRAS, 208, 15

Smith, D. A., \& Done, C. 1996, MNRAS, 280, 355

Turner, T. J., George, I. M., Nandra, K., \& Mushotzky, R. F. 1997, ApJ, 493, 91

Turner, T. J., \& Pounds, K. A. 1989, MNRAS, 240, 833 